\title{
A Randomized, Double-Blind, Placebo-Controlled Trial: The Efficacy of Multispecies Probiotic Supplementation in Alleviating Symptoms of Irritable Bowel Syndrome Associated with Constipation
}

\author{
Valerio Mezzasalma, ${ }^{1}$ Enrico Manfrini, ${ }^{1}$ Emanuele Ferri, ${ }^{1}$ \\ Anna Sandionigi, ${ }^{1}$ Barbara La Ferla, ${ }^{1}$ Irene Schiano, ${ }^{2}$ Angela Michelotti, ${ }^{2}$ \\ Vincenzo Nobile, ${ }^{2}$ Massimo Labra, ${ }^{1}$ and Patrizia Di Gennaro ${ }^{1}$ \\ ${ }^{1}$ Department of Biotechnology and Biosciences, University of Milano-Bicocca, Piazza della Scienza 2, 20126 Milan, Italy \\ ${ }^{2}$ Farcoderm Srl., Via Angelini 21, San Martino Siccomario, 27028 Pavia, Italy
}

Correspondence should be addressed to Patrizia Di Gennaro; patrizia.digennaro@unimib.it

Received 22 March 2016; Revised 28 June 2016; Accepted 3 July 2016

Academic Editor: Clara G. de los Reyes-Gavilan

\begin{abstract}
Copyright (C) 2016 Valerio Mezzasalma et al. This is an open access article distributed under the Creative Commons Attribution License, which permits unrestricted use, distribution, and reproduction in any medium, provided the original work is properly cited.
\end{abstract}

Background and Aim. The efficacy of supplementation treatment with two multispecies probiotic formulates on subjects diagnosed with IBS-C and the assessment of their gut microbiota were investigated. Methods. A randomized, double-blind, three-arm parallel group trial was carried out on 150 IBS-C subjects divided into three groups (F_1, F_2, and F_3). Each group received a daily oral administration of probiotic mixtures (for 60 days) F_1 or F_2 or placebo F_3, respectively. Fecal microbiological analyses were performed by species-specific qPCR to assess the different amount of probiotics. Results. The percentage of responders for each symptom was higher in the probiotic groups when compared to placebo group during the treatment period $(t 60)$ and was maintained quite similar during the follow-up period $(t 90)$. Fecal analysis demonstrated that probiotics of the formulations increased during the times of treatment only in fecal DNA from subjects treated with F_1 and F_2 and not with F_3, and the same level was maintained during the follow-up period. Conclusions. Multispecies probiotic supplementations are effective in IBS-C subjects and induce a different assessment in the composition of intestinal microbiota. This clinical study is registered with the clinical study registration number ISRCTN15032219.

\section{Introduction}

The intestinal microbiota consists of a wide range of bacterial species [1]. The microbial community resides in the gut of the host establishing a mutually beneficial relationship and modulating, through its metabolic activities, the host's health status $[2,3]$. The microbiota exerts different physiological functions such as inhibition of pathogenic bacteria and synthesis of short fatty acids; stimulation of nutrient and mineral absorption, as well as modulation of the intestinal immune system; synthesis of vitamins and amino acids; and the decomposition of protein compounds $[4,5]$. The intestinal microbiota in healthy adults is generally considered stable over time in predominance of bacteria belonging to four main phyla: Bacteroidetes and Firmicutes as prevalent and Actinobacteria and Proteobacteria that are less represented. The microbiota in subjects with Irritable Bowel Syndrome (IBS) has been shown to be less stable compared to healthy adults $[5,6]$. A controlled balance between bacterial species considered beneficial (lactobacilli and bifidobacteria) associated with the reduction of those considered harmful (Clostridium, E. coli, Salmonella, and Pseudomonas) is fundamental for maintenance of the gut physiological state and should attenuate IBS symptoms. Alteration of the microbiota's composition, which can be caused by psychophysical, dietary, or environmental stress, is defined as dysbiosis and can lead 
to pathological conditions. Moreover, aberrant stimulation of the immune system leading to inflammation may be reliably encountered as the potential link between dysbiosis and metabolic diseases [7-9].

Experimental evidences have increasingly shown a correlation between microbiota imbalance and the induction of Irritable Bowel Syndrome. In particular, dysbiosis was proven to underlie a modification of intercellular tight junctions, responsible under normal conditions for the correct structure of the epithelial layer of intestinal mucosa, which is known to increase the mucosal permeability. Consequently, antigens have been detected in the intercellular space with the activation of the inflammatory cascade, production of cytokines, and tissue damage [9-11].

Oral administration of appropriate probiotic strains may therefore be beneficial to health and in particular with respect to IBS. However, clinical data reporting changes in the detection of ingested probiotic traceability in the gastrointestinal tract of patients and the related effects on the gut are still few and inconclusive [12-15]. Studies have reported an improvement in global symptoms with probiotics, while others have failed to demonstrate any benefit [16-18].

Moreover, it is widely known that the effect of probiotics is species-specific $[19,20]$. These indications combined with the diversity and complexity of IBS may indicate that a probiotic combination could be more efficient than a single strain in this particular syndrome. Some authors suggested that multispecies probiotics may in some conditions be more efficient than monospecies probiotics due to, for example, enhanced intestinal adhesion and the production of a greater variety of antimicrobial compounds, compared with single probiotic [15].

In this regard, the present study was aimed at testing different probiotics, which we had previously characterized [19] for their in vitro activities, in order to demonstrate by means of in vivo detection procedures their distinctive effects on the intestine after oral administration. The aim of this randomized double-blind, placebo-controlled study was to investigate the efficacy of two probiotic formulations in ameliorating IBS-C subjects. The effects of probiotics on IBS symptoms in comparison with placebo and their assessment in the gut microbiota after probiotic therapy by analysing fecal bacteria were evaluated. In order to reach this goal, 150 volunteer subjects were recruited and included in three groups (F_1, F_2, and F_3). Each group received 60-day oral administration of different probiotic mixtures: F_1 containing L. acidophilus and L. reuteri; F_2 containing L. plantarum, L. rhamnosus, and B. animalis subsp. lactis; and F_3 containing placebo. At different time points after probiotic oral administration, fecal microflora was analysed by real-time quantitative PCR performed on DNA from stool samples of the subjects. An additional 30-day followup period was encountered in order to assess whether the observed beneficial effects still persist after treatment suspension.

Our investigation provides a relevant contribution to investigate the efficacy of multispecies probiotics in treating IBS-C and other gastrointestinal syndromes related to the gut disorders.

\section{Materials and Methods}

2.1. Study Design. This randomized, double-blind study was carried out in accordance with the Declaration of Helsinki and the Good Clinical Practice guidelines E6. The study protocol and the informed consent form were approved by the "Independent Ethical Committee for Non-Pharmacological Clinical studies" during its meeting on July 17, 2013. All subjects provided a written informed consent before initiation of any study-related procedures. The study took place at Farcoderm Srl. facilities. Farcoderm Srl. is an independent testing laboratory for in vitro and in vivo safety and efficacy assessment of cosmetics, food supplements, and medical devices.

Enrolled subjects were randomly assigned to receive one capsule of the two different formulations of probiotics (mix F_1 and mix F_2) or placebo (mix F_3) once daily for a period of 60 days and were followed up for a further period of 30 days after a follow-up period from the last ingestion of the tested products.

The tested products consisted of food supplements (capsules) containing lactobacilli and bifidobacteria (Principium Europe Srl., Solaro, MI, Italy) (Table 1). The composition of the probiotic mix F_1 was as follows: $5 \times 10^{9} \mathrm{CFU} L$. acidophilus (30 $\mathrm{mg}$ as lyophilized), $5 \times 10^{9} \mathrm{CFU}$ L. reuteri (30 $\mathrm{mg}$ as lyophilized), $330 \mathrm{mg}$ inulin, $5 \mathrm{mg}$ silica, and $5 \mathrm{mg}$ talc. The F_2 composition was as follows: $5 \times 10^{9} \mathrm{CFU} L$. plantarum (12 mg as lyophilized), $5 \times 10^{9} \mathrm{CFU}$ L. rhamnosus (20 mg as lyophilized), $5 \times 10^{9} \mathrm{CFU}$ B. animalis subsp. lactis (60 $\mathrm{mg}$ as lyophilized), $298 \mathrm{mg}$ inulin, $5 \mathrm{mg}$ silica, and $5 \mathrm{mg}$ talc. Placebo (F_3) composition was as follows: $390 \mathrm{mg}$ inulin, $5 \mathrm{mg}$ silica, $5 \mathrm{mg}$ talc.

The study flow and the schedule of assessment chart is reported in Figure 1. A questionnaire and an explanation of the protocol of the study were given to the subjects. Symptom questionnaire was performed as an interview to the enrolled subjects at the time points of the study (each 10 days).

Stool samples for fecal microbiota analysis were obtained at the start of the treatment $(t 0)$ and at times $t 10, t 30$, and $t 60$ days during the treatment for a total period of 60 days, followed by a sample at $t 90$ days after washing up for 30 days, for a total period of 90 days from the start of the treatment.

2.2. Subjects of the Study. Eligible subjects were all adult males and females aged between 18 and 65 years suffering from Irritable Bowel Syndrome with constipation (IBS-C) diagnosed by clinical analyses and self-reported interviews. Subjects suffering from IBS-C were screened by means of the Rome III diagnostic criteria questionnaire [21]. Exclusion criteria were (i) pregnancy or the intention to become pregnant, (ii) lactation, (iii) food intolerances/allergy, (iv) known history of other gastrointestinal disorders, (v) chronic or acute gastrointestinal disorders, (vi) participation in another similar study, and (vii) unwillingness or inability to comply with the study protocol requirements.

The study further excluded subjects using food supplements (included probiotics different from the study) or drugs containing actives having an influence on gastrointestinal 
TABLE 1: List of the strains used in this study, deposit number, and the most relevant antimicrobial activities described in Presti et al. 2015 [19]. Abbreviations used in the present work were also included.

\begin{tabular}{|c|c|c|c|}
\hline Probiotic strain & Deposit number & Antimicrobial activity $v s$ & Abbr. \\
\hline $\begin{array}{l}\text { Lactobacillus rhamnosus LRH020 } \\
\text { (formerly PBS070) }\end{array}$ & DSM 25568 & C. albicans; E. faecalis; P. aeruginosa; S. aureus; E. coli & L_Rha \\
\hline Lactobacillus plantarum PBS067 & DSM 24937 & C. albicans; E. faecalis; P. aeruginosa; S. aureus; E. coli & L_Pla \\
\hline $\begin{array}{l}\text { Bifidobacterium animalis subsp. lactis } \\
\text { BL050 (formerly PBS075) }\end{array}$ & DSM 25566 & E. faecalis; P. aeruginosa; E. coli & B_Lac \\
\hline Lactobacillus acidophilus PBS066 & DSM 24936 & C. albicans; E. faecalis; P. aeruginosa; S. aureus; E. coli & L_Aci \\
\hline Lactobacillus reuteri $\mathrm{PBS} 072$ & DSM 25175 & E. faecalis & L_Reu \\
\hline
\end{tabular}

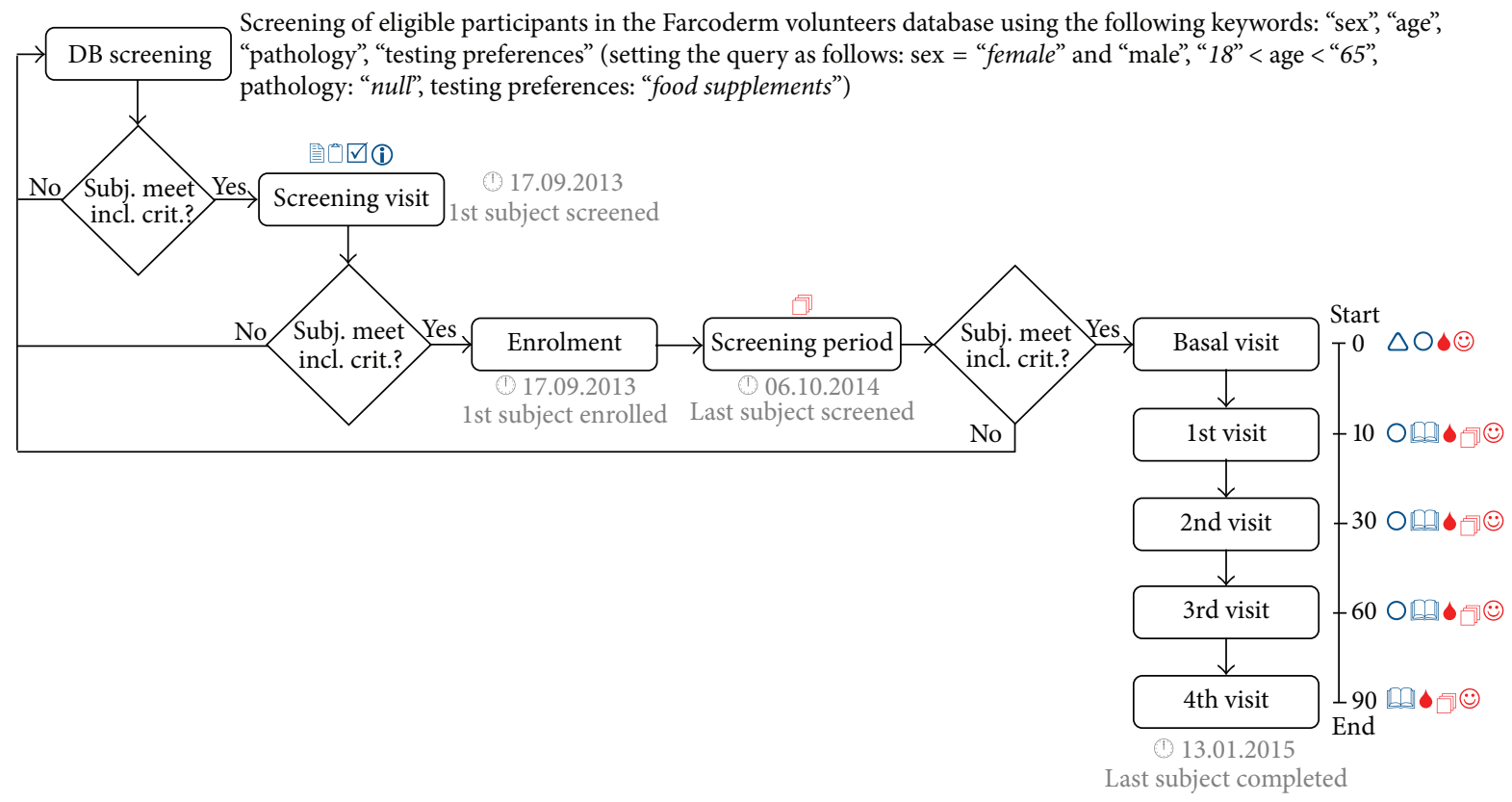

Study-related procedures

祭 Informed consent signature

Medical examination

$\checkmark$ Eligibility check

$\triangle$ Randomization (i) Rome III diagnostic criteria

O Product intake

[D] Alimentary diary
Endpoints

- Stool collection

7. Symptoms questionnaire

(:) Quality of life (QoL)

FIgURE 1: Study flow and schedule of assessment chart.

physiology. Changes in diet or lifestyle were not allowed during all the study period.

\subsection{Assessment of Clinical Effects}

2.3.1. Endpoints. Patients were evaluated five times during the course of the study: at baseline, after 10,30, and 60 days from the start period, and 30 days after the follow-up of the intervention period to assess postintervention effects. Primary efficacy endpoints were the proportion of participants whose IBS symptoms after probiotic supplementations were relieved up to 60 days and the assessment of their gut microbiota. The secondary efficacy endpoint was the maintenance of the obtained effects 30 days after the last product(s) intake.
2.3.2. Symptoms Questionnaire. IBS-C related symptoms of subjects were reported daily by a questionnaire according to Guide Lines of FDA (Guidance for Industry-Irritable Bowel Syndrome-Clinical Evaluation of Drugs for Treatment). IBSC questionnaire consisted of 5 items as follows: (i) bloating, (ii) abdominal pain, (iii) constipation, (iv) abdominal cramps, and (v) flatulence. For each item subjects scored the symptom severity on a 10-point Visual Analogue Scale (VAS). Data are reported as the mean values after every 10 days.

\subsubsection{Health-Related Quality of Life (HR-QOL). HR-QOL} was assessed by means of the Italian version of the Quality of Life Measure for Persons with IBS [22]. From the original 34 items questionnaire, the following 12 items were selected: (i) I 
TABLE 2: List of primers used in this study.

\begin{tabular}{|c|c|c|c|c|}
\hline Probiotic & Primer code & Sequence $\left(5^{\prime} \rightarrow 3^{\prime}\right)$ & DNA region & $\begin{array}{c}\text { Amplified } \\
\text { length (bp) }\end{array}$ \\
\hline \multirow{2}{*}{ L. rhamnosus } & LraF & CTAGCGGGTGCGACTTTGTT & \multirow{2}{*}{$16 S / 23 S$ IS } & \multirow{2}{*}{$123 \mathrm{bp}$} \\
\hline & LraR & CAGCGGTTATGCGATGCGAA & & \\
\hline \multirow{2}{*}{ L. plantarum } & Lpl2F & CATTGGAACCGAACCAGTTG & \multirow{2}{*}{$16 S / 23 S$ IS } & \multirow{2}{*}{$203 \mathrm{bp}$} \\
\hline & Lpl2R & CGGTGTTCTCGGTTTCATTATG & & \\
\hline \multirow{2}{*}{ B. animalis subsp. lactis } & AnimF & GCACGGTTTTGTGGCTGG & \multirow{2}{*}{ pre16S } & \multirow{2}{*}{$171 \mathrm{bp}$} \\
\hline & AnimR & GACCTGGGGGACACACTG & & \\
\hline \multirow{2}{*}{ L. acidophilus } & Lacid2F & GGGCAAATCACGAACGAGTA & \multirow{2}{*}{ pre16S } & \multirow{2}{*}{$132 \mathrm{bp}$} \\
\hline & Lacid2R & CTTTGTTTTCGTTCGCTTCA & & \\
\hline \multirow{2}{*}{ L. reuteri } & Lreu2F & GTTGACGAAAGAATGAAATCCA & \multirow{2}{*}{ pre16S } & \multirow{2}{*}{$118 \mathrm{bp}$} \\
\hline & Lreu2R & TCATGTCGTCAATCAGATGTCA & & \\
\hline
\end{tabular}

am embarrassed by the smell caused by my bowel problems, (ii) I feel vulnerable to other illnesses because of my bowel problems, (iii) I feel fat because of my bowel problems, (iv) I feel my life is less enjoyable because of my bowel problems, (v) I feel depressed about my bowel problems, (vi) I have to watch the amount of food I eat because of my bowel problems, (vii) because of my bowel problems, sexual activity is difficult for me, (viii) I feel angry that I have bowel problems, (ix) I feel I get less done because of my bowel problems, (x) my bowel problems limit what I can wear, (xi) I have to watch the kind of food I eat because of my bowel problems, and (xii) I fear that I won't be able to have a bowel movement.

For each item, the following five-point response scale was used: 1: not at all, 2: slightly, 3: moderately, 4: quite a bit, and 5: extremely. HR-QOL was interviewer-administered.

2.4. Fecal Samples (Stools Type Classification). Fecal samples were collected from subjects at $t 10, t 30, t 60$, and $t 90$ time points during the study. Fresh fecal samples were homogenized by vortex mixing of the fecal mass and separated into aliquots to be stored at $-80^{\circ} \mathrm{C}$ until the analysis using DNAbased method quantitative PCR.

Stool types were classified according to Bristol scale [23]. Bristol values were divided in two categories: values 3 (sausage shape with cracks in the surface, normal), 4 (smooth soft sausage or snake, normal), and 5 (soft blobs with clear-cut edges, lacking fiber), corresponding to healthy bowel situation, were assigned to class 1, whereas values 1 (separate hard lumps, very constipated), 2 (lumpy and sausage like, slightly constipated), 6 (mushy consistency with ragged edges, inflammation), and 7 (liquid consistency with no solid pieces, inflammation) were assigned to class 0 . The number of volunteer subjects with stool samples with Bristol values belonging to class 1 was calculated for each treatment and for each experimental time.

2.5. Probiotic Strains and DNA Extraction. In this study, a total of four Lactobacillus spp. strains and one Bifidobacterium strain, supplied from a private collection, were taken into consideration for the preparation of the two formulations (F_1 and F_2). Table 1 describes the characteristics of each strain. In order to prepare standard curves of DNA extracted from probiotics, microbial cultures were performed in MRS (Conda) medium and incubated at $37^{\circ} \mathrm{C}$ for 24 hours in anaerobic conditions using anaerobic atmosphere generation bags (Anaerogen, Oxoid). For B. animalis subsp. lactis, a supplementation of $0.3 \mathrm{~g} / \mathrm{L}$ L-cysteine hydrochloride monohydrate was included in the growth medium (cMRS) (SigmaAldrich).

DNA from microbial cultures was extracted by the ZR fecal DNA MiniPREP (Zymo Research). A total of $1 \mathrm{~mL}$ of $10^{9} \mathrm{CFU} / \mathrm{mL}$ culture was used for obtaining genomic DNA following the protocol provided by the manufacturer.

DNA extraction from stool samples was performed from $150 \mathrm{mg}$ of feces by the ZR fecal DNA MiniPREP. Both DNA extracted from probiotic cultures and DNA from stool samples were utilized to perform qPCR.

2.6. Fecal Microbiology Analysis by Quantitative PCR. qPCR reactions were carried out using an ABI 7500 (Applied Biosystems) and the SsoFast EvaGreen Supermix with Low ROX (BIO-RAD) dye. We designed species-specific primer sets developed by the authors in a previous study and reported in Table 2 [24]. Reactions were carried out in a $10 \mu \mathrm{L}$ qPCR mix containing $5 \mu \mathrm{L}$ of SsoFast EvaGreen Supermix with Low ROX, $0.2 \mu \mathrm{L}$ of $10 \mu \mathrm{M}$ forward primer and $10 \mu \mathrm{M}$ reverse primer, $4 \mu \mathrm{L}$ of DNA template, and $2.4 \mu \mathrm{L}$ of $\mathrm{H}_{2} \mathrm{O}$, according to the following qPCR program: $10^{\prime} 95^{\circ} \mathrm{C}$ and 40 cycles of $15^{\prime \prime}$ $95^{\circ} \mathrm{C}$ and $1^{\prime} 60^{\circ} \mathrm{C}$ (followed by a dissociation step).

For each strain, standard curves were constructed using DNA extracted from microbial cultures using tenfold dilutions ranging from $10^{8} \mathrm{CFU} / \mathrm{mL}$ to $10 \mathrm{CFU} / \mathrm{mL}$. Each DNA sample both from feces and from culture dilution was analysed in triplicate.

2.7. Sample Size. Sample size was calculated with a twoside $5 \%$ significance level and a power of $80 \%$ taking into account a $23 \mathrm{~mm}$ margin of equivalence among treatments. A sample size of 50 subjects per treatment arm was considered necessary given an anticipated dropout rate of $40 \%$. Taking into consideration the duration of the treatment and the 
complexity of the inclusion, we expected a high rate of dropout.

2.8. Randomization. Subjects were assigned to treatment arms using a computer-generated PASS 11 statistical software (version 11.0.8 for Windows; PASS, LLC, Kaysville, UT, USA) restricted randomization list ("Efron's biased coin" algorithm). Subjects were randomized in a $1: 1: 1$ (F_1:F_2:F_3) ratio. The software was running on Windows Server 2008 R2 Standard SP1 64 Edition (Microsoft, USA). Subjects, investigator, and collaborators were kept blind to products assignment. The randomization list was stored in a safe place by the in site study director.

2.9. Statistical Methods. Statistical analysis was performed using NCSS 8 (version 8.0.4 for Windows; NCCS, LLC) running on Windows Server 2008 R2 64 Edition. Internal consistency was checked before statistical analysis in order to assess subject's reliability. For IBS-C related symptoms, the number of responders to treatment was calculated. A responder was defined as the subject reporting a decrease of symptoms of at least $30 \%$ compared to the basal condition for at least $50 \%$ of the intervention time (Guidance for IndustryIrritable Bowel Syndrome-Clinical Evaluation of Drugs for Treatment). Positive/negative responses to treatment/placebo were tested using Fisher's exact ratio test. HR-QOL and follow-up data were submitted to RM-ANOVA followed by Tukey-Kramer posttest. Data normality was checked using skewness, kurtosis, and omnibus test. Statistical significance was reported as follows: ${ }^{*} P<0.05,{ }^{* *} P<0.01$, and ${ }^{* * *} P<$ 0.001 .

In order to apply generalized linear mixed model (GLMM) under Poisson-lognormal error to account for higher variation at the lower end of target abundance, MCMC.qPCR R package [25] was used to convert Ct data in bacterial counts. The conversion to approximate counts uses the following formula:

$$
\text { Count: } E^{(\mathrm{Ct} 1-\mathrm{Ct})} \text {, }
$$

where $E$ is the efficiency of amplification and Ct1 is the number of qPCR cycles required to detect a single target molecule.

Markov Chain Monte Carlo (MCMC) algorithm implemented in the package is used to sample from the joint posterior distribution over all model parameters, in order to estimate the effects of all experimental factors on the levels of specific microbial species. GLMM was used to test whether the levels of the different microbial species in different formulation groups (F_1, F_2, and F_3) differed between the baseline $(t 0)$ and the subsequent time points $(t 10, t 30, t 60$, and $t 90)$.

The experimental design is incorporated into the following model:

$\ln ($ counts $) \sim$ species + species:Formulation + species:Time + sample + species:sample + species:residual,

where the logarithm of bacterial counting rate is the variable response and the fixed factors are Formulation and
TABle 3: Demographic and baseline characteristics of the subjects of the clinical study ${ }^{*}$. Data are mean \pm SE.

\begin{tabular}{lccc}
\hline & F_1 & F_2 & F_3 \\
\hline Number of subjects & 50 & 50 & 50 \\
Age & $36.0 \pm 11.9$ & $38.0 \pm 12.1$ & $38.1 \pm 13.5$ \\
Bloating (VAS) & $6.3 \pm 0.2$ & $6.2 \pm 0.2$ & $6.1 \pm 0.2$ \\
Abdominal pain & $5.0 \pm 0.2$ & $4.9 \pm 0.2$ & $4.8 \pm 0.2$ \\
Constipation & $6.6 \pm 0.1$ & $6.5 \pm 0.1$ & $6.1 \pm 0.2$ \\
Abdominal cramps & $4.2 \pm 0.2$ & $3.9 \pm 0.2$ & $4.1 \pm 0.2$ \\
Flatulence & $4.6 \pm 0.3$ & $4.4 \pm 0.2$ & $4.4 \pm 0.2$ \\
\hline
\end{tabular}

${ }^{*}$ There were no statistically significant differences between the three groups.

Time (baseline and subsequent time points). The three remaining factors sample (different subjects of the study), species:sample, and species:residual are defined as random factors, accounting for the variation in quality and quantity of biological material among samples. [26].

To produce graphical chart, we used ggplot $2 \mathrm{R}$ package

\section{Results}

3.1. Subjects of the Study. The study was conducted between September 2013 and January 2015. A total of 157 male and female subjects suffering from IBS-C were successfully enrolled (Figure 2). Subjects were randomized to active or placebo treatments as follows: (i) 53 subjects were randomized to F_1, (ii) 52 subjects were randomized to F_2, and (iii) 52 subjects were randomized to F_3. Seven subjects discontinued intervention because they were no longer interested in participating in the study.

After randomization, subjects attended four clinic visits every month, except for the first visit (10 days after product use). The population was Caucasian and the mean $( \pm S D)$ age was $37.4 \pm 12.5$ years. Demographic and baseline characteristics (Table 3) were similar across treatment arms, indicating an unbiased randomization. The per-protocol population consisted of 150 subjects. All subjects were included in the safety analysis dataset.

3.2. Primary Efficacy Endpoint. The results of IBS-C related symptoms amelioration of the responder are reported in Figure 3. The number of responders to treatment was defined as the subject reporting a decrease of symptoms of at least $30 \%$ compared to the basal condition for at least $50 \%$ of the intervention time. Internal consistency for each item, over time, was good (Cronbach's alpha $>0.88$ ). The percentage of responders for each clinical symptom was higher in the F_1 and F_2 group when compared to placebo F_3 (F_1 versus F_3, in the range of $66 \%-78 \%$ versus $6 \%-36 \%$; and F_2 versus $F_{-} 3$ in the range of $78 \%-90 \%$ versus $\left.6 \%-36 \%\right)(P<$ $0.001)$. Neither statistical nor clinically significant differences were detected between F_1 and F_2 except for constipation symptom which was less significant during the treatment $(P<0.01)$. 


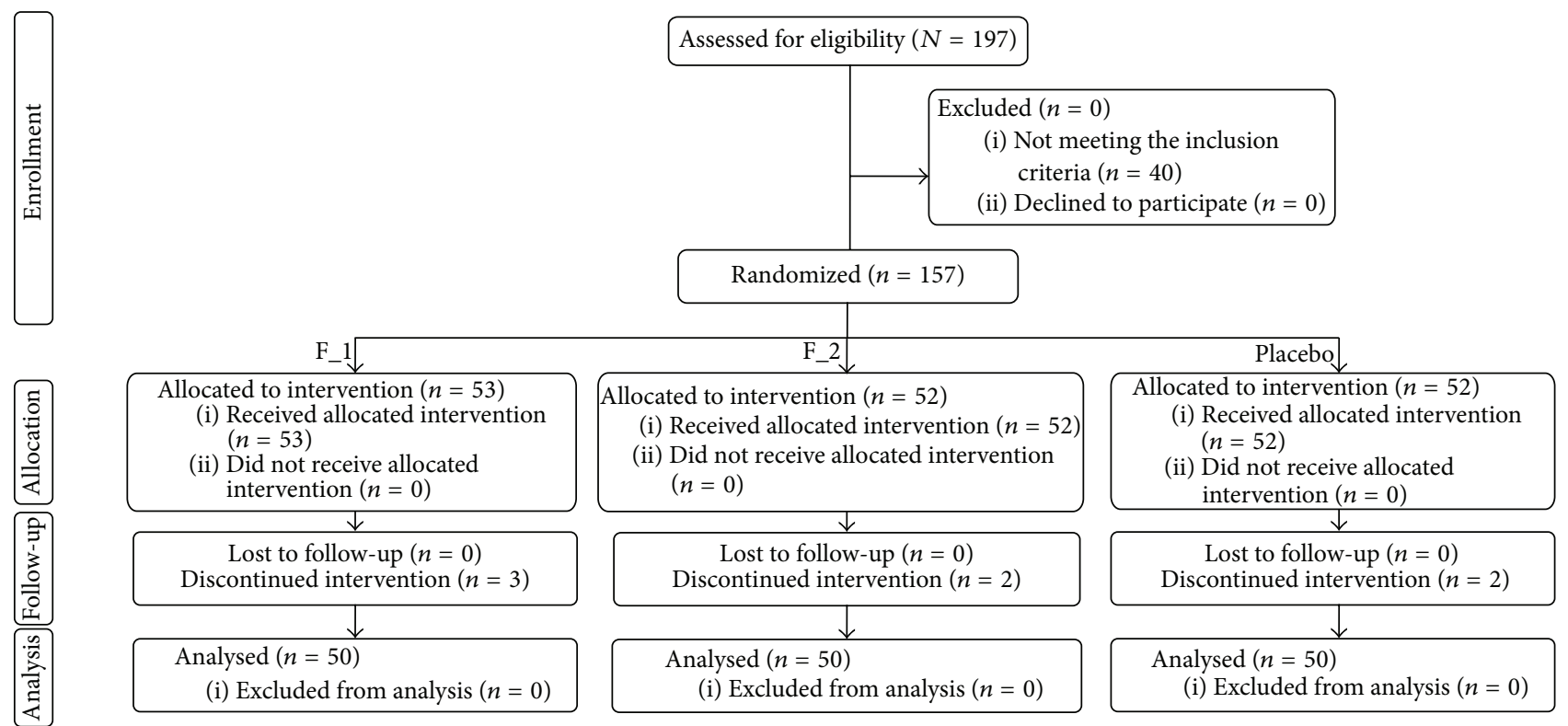

Figure 2: Disposition of the subjects of the study.

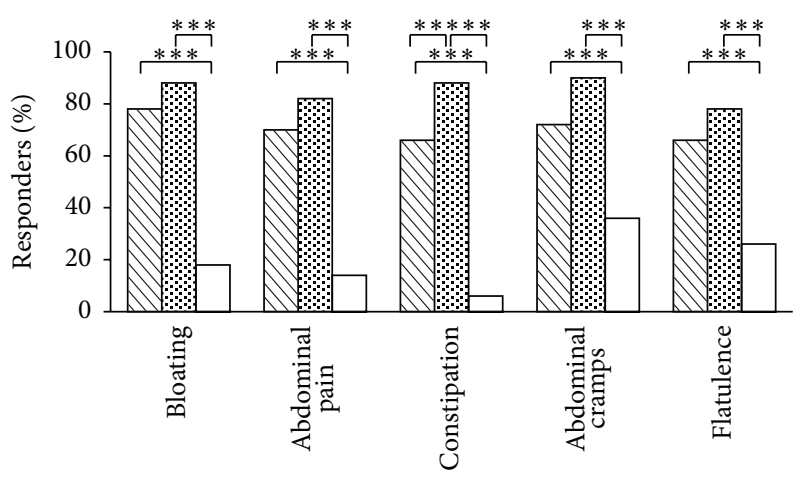

$\mathbb{Q}$ F_1

F 82

$\square$ F_3

FIgURE 3: Percentage of responders to IBS-C related symptom during the treatment period ( $t 60$, days) with probiotic formulations F_1 and F_2. The Responders was defined as the subject reporting a decrease of symptoms of at least $30 \%$ compared to the basal condition for at least $50 \%$ of the intervention time. Bloating, abdominal pain, constipation, abdominal cramps, and flatulence symptoms were assessed on a numbering scale from 0 to 10 for each item subjects scored. Data are mean \pm SE. Upon the square brackets are reported the intergroups F_1 and F_2 (versus placebo F_3) statistical analysis $\left({ }^{* * *} P<0.001\right)$. The intergroups $F_{-} 1$ versus F_2 statistical analysis $\left({ }^{* *} P<0.01\right)$ is also reported.

The results of HR-QOL are reported in Table 4. Data were reported as the sum of each score given by the subject to each item of the HR-QOL questionnaire. Internal consistency for each item, over time, was good (Cronbach's alpha $>0.86$ ). The HR-QOL was ameliorated for subjects treated with both F_1 and F_2 during the treatment period. Relatively to baseline, the sum of each score given by the subject to each symptom during the treatment ( $t 60)$ was significantly reduced in the probiotics group $(31.2 \pm 1.0 \rightarrow 20.2 \pm 0.9, P<0.001$, for F_1 group; and $32.0 \pm 0.9 \rightarrow 20.4 \pm 0.9, P<0.001$, for F_2 group), but not so clinically relevant in the placebo group $\left(30.0 \pm 0.9 \rightarrow 26.9 \pm 1.2, P<0.001\right.$, for $F_{-}$3 group). As expected, mild amelioration of HR-QOL was seen in the placebo-treated subjects, probably due to the placebo effect. The intergroup amelioration of HR-QOL during the treatment ( $t 60)$ was bigger in the actives-treated (F_1 and F_2) subjects compared to placebo-treated (F_3) subjects (F_1 versus $F_{\_} 3$, $20.2 \pm 0.9$ versus $26.9 \pm 1.2$, and $F_{2} 2$ versus $F_{\_}$, $20.4 \pm 0.9$ versus $26.9 \pm 1.2, P<0.001)$. Neither statistical nor clinically significant differences were found between $F_{-} 1$ and F_2.

In addition, the analysis of the stool type classification was performed (data not shown). The numbers of samples with values of 3,4 , and 5, according to Bristol scale and representing a healthy bowel movement were calculated. Significant differences were observed between F_1 or F_2 with respect to F_3, representing an increase in the number of stool samples with a healthier characteristic in the probiotic treated groups.

3.3. Secondary Efficacy Endpoint. In order to assess the maintenance of the obtained effects, the study design foresaw a further 30-day follow-up period after the 60-day product intake period. The results of IBS-C related symptoms maintenance for responder are reported in Figure 4. During the follow-up period (from day 61 to day 90), the percentage of responders for each clinical symptom was higher in the F_1 and $\mathrm{F} \_2$ groups when compared to placebo $\mathrm{F} \_3$ (F_1 versus F_3, in the range of $56 \%-74 \%$ versus $10 \%-40 \%$; and F_2 versus F_3 in the range of $76 \%-82 \%$ versus $10 \%-40 \%)(P<0.001)$. Neither statistical nor clinically significant differences were detected between F_1 and F_2 except for abdominal cramps 
TABLE 4: HR-QOL amelioration at the different times of treatment $(t 0, t 10, t 30$, and $t 60$, days) and at the follow-up period, that is, 30 days after the last product intake ( $t 90$, days) between F_1 and F_2 groups compared with F_3. Bloating, abdominal pain, constipation, abdominal cramps, and flatulence were assessed on a numbering scale from 0 to 10 for each item subjects scored. Data are mean \pm SE. In brackets is reported the intergroups (versus placebo) statistical analysis.

\begin{tabular}{lcccccccc}
\hline Time (days) & F_1 & $P$ value & F_2 & $P$ value & F_3 & $P$ value & F_1 vs F_3 $(P$ value $)$ & F_2 vs F_3 $(P$ value $)$ \\
\hline 0 & $31.2 \pm 1.0$ & & $32.0 \pm 0.9$ & & $30.0 \pm 0.9$ & & & \\
10 & $28.3 \pm 0.9$ & $<0.01$ & $27.0 \pm 0.8$ & $<0.001$ & $28.9 \pm 1.0$ & $>0.05$ & $>0.05$ & $>0.05$ \\
30 & $23.1 \pm 0.9$ & $<0.001$ & $22.8 \pm 0.8$ & $<0.001$ & $27.8 \pm 1.1$ & $<0.05$ & $<0.01$ & $<0.001$ \\
60 & $20.2 \pm 0.9$ & $<0.001$ & $20.4 \pm 0.9$ & $<0.001$ & $26.9 \pm 1.2$ & $<0.001$ & $<0.001$ & $<0.001$ \\
90 & $22.2 \pm 1.0$ & $<0.001$ & $22.0 \pm 0.8$ & $<0.05$ & $28.7 \pm 1.2$ & $<0.01$ & $<0.001$ & $<0.001$ \\
\hline
\end{tabular}

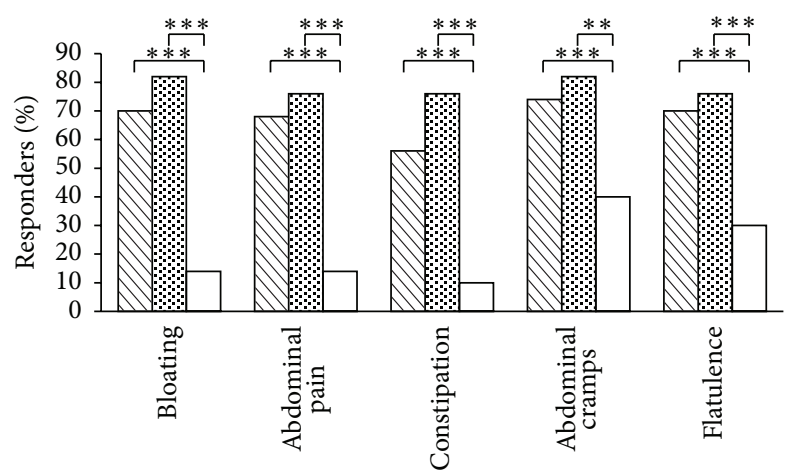

$\mathbb{B}$ F_1

F_2

$\square$ F_3

FIgURE 4: Percentage of responders to IBS-C related symptom at the follow-up period, that is, 30 days after the last product intake ( $t 90$, days) of probiotic formulations F_1 and F_2. The Responders was defined as the subject reporting a decrease of symptoms of at least $30 \%$ compared to the basal condition for at least $50 \%$ of the intervention time. Bloating, abdominal pain, constipation, abdominal cramps, and flatulence symptoms were assessed on a numbering scale from 0 to 10 for each item subjects scored. Data are mean \pm SE. Upon the square brackets are reported the intergroups F_1 and F_2 (versus placebo F_3) statistical analysis $\left({ }^{* * *} P<0.001\right)$. The intergroups F_1 versus F_2 statistical analysis $\left({ }^{* *} P<0.01\right)$ is also reported.

symptom which was less significant during the follow-up period $(P<0.01)$.

The results of HR-QOL are reported in Table 4. Data were reported as the sum of each score given by the subject to each item of the HR-QOL questionnaire. Data obtained 30 days (t90) after the last product intake were lower in the probiotic groups with respect to the basal scoring of symptoms at $t 0$ $(31.2 \pm 1.0 \rightarrow 22.2 \pm 1.0, P<0.001$, for F_1 group; and $32.0 \pm$ $0.9 \rightarrow 22.0 \pm 0.8, P<0.05$, for $\mathrm{F} \_2$ group), but not so clinically relevant in the placebo group $(30.0 \pm 0.9 \rightarrow 28.7 \pm 1.2, P<$ 0.01 , for F_3 group).

The intergroup amelioration of HR-QOL was bigger in the actives-treated (F_1 and F_2) subjects compared to placebo-treated (F_3) subjects $(22.2 \pm 1.0$ versus $28.7 \pm 1.2$ and $22.0 \pm 0.9$ versus $28.7 \pm 1.2, P<0.001)$. Neither statistical nor clinically significant differences were detected between F_1 and F_2.
Comparing follow-up period ( $t 90$ ) with the end of treatment period $(t 60)$, the HR-QOL was not significantly different for both F_1 and F_2 probiotic groups, indicating the maintenance of the obtained effects.

3.4. Fecal Microbiology Analysis by Quantitative PCR. DNA was extracted from fecal samples of the subjects enrolled in this study at the times $t 0, t 10, t 30, t 60$, and $t 90$ from the first ingestion of the probiotic formulations. The qPCR analysis demonstrated that the species-specific sequences associated with the probiotics of the formulations were detected only in fecal DNA from subjects treated with the formulations F_1 and F_2 and not with the formulation F_3 and that no significant difference was detected between the two kinds of formulations.

The qPCR assay for L. plantarum and L. rhamnosus (contained in the mix F_2) demonstrated a quite similar quantity of these probiotic bacteria during the times of treatment, while B. animalis subsp. lactis decreases at time 90 after the follow-up period. Concerning results of probiotics contained in the formulation F_1, we can observe that $L$. acidophilus increases during the treatment but decreases after the follow-up period, while the quantity of $L$. reuteri was quite similar during all the period of treatment including time t90 (Figure 5). All these results indicate that all probiotics utilized in this study were enhanced in the gut tract after their ingestion at least for 90 days; the only exception was observed for B. animalis subsp. lactis in which a lower concentration of this probiotic in the postintervention samples was obtained.

\section{Discussion}

Probiotics exert their actions through interaction with host intestinal cells. Their supplementation significantly modifies the intestinal microbiota by increasing lactobacilli and bifidobacteria that can improve through the combination with specific probiotics providing a health benefit to the host [27]. Multispecies probiotics may have a variety of different beneficial effects particularly on IBS symptoms because each species acts in a particular way on the gastrointestinal tract, and two or more species acting together may have a synergistic effect [15].

Although several trials have demonstrated the superiority of probiotics (above all, lactobacilli and bifidobacteria) over placebo in controlling IBS symptoms [16-18], however, given 
F_1
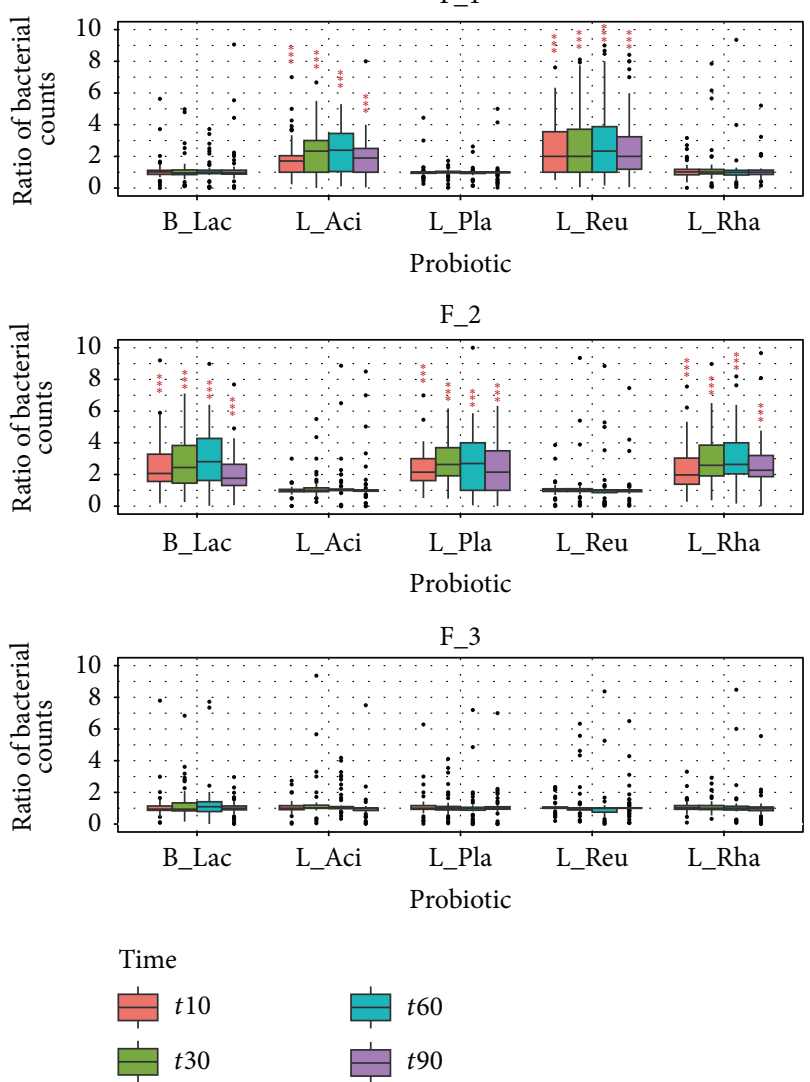

Figure 5: Ratio of probiotics of formulations (F_1 and F_2, versus F_3) by qPCR of species-specific sequences at the different times of treatment versus the amount at the baseline time point, expressed as bacterial counts. Upon the bars is reported the statistical analysis between treatments $\left({ }^{* * *} P<0.001\right)$.

the controversies in IBS pathophysiology or lack of clear evidence for gut microbiota abnormalities in patients with IBS, additional randomized clinical trials with appropriate endpoints and design are needed to evaluate to which extent probiotics are a useful therapeutic strategy in the management of IBS symptoms.

In this study, a randomized, double-blind, placebocontrolled clinical trial with two formulations containing different probiotics was developed and the evaluation of gut microbiota assessment and gastrointestinal benefits of IBS-C patients was determined until 90 days. Multispecies probiotics were used for the treatment of IBS-C in our study: $L$. acidophilus, $L$. reuteri, $L$. plantarum, L. rhamnosus, and $B$. animalis subsp. lactis. Indeed, it is known that the level of bifidobacteria and lactobacilli species is lower in IBS patients compared to healthy persons $[28,29]$ and several studies show that the supplementation of them, or mixtures including species of these genera, is effective in alleviating symptoms of IBS. Moreover, the selected strains were already known for their effect on intestinal cell lines as previously reported [19].

To investigate the alterations in the intestinal microbiota, the number of lactobacilli and bifidobacteria present in fecal samples of recruited subjects was determined by quantitative real-time PCR. The numbers of Lactobacillus spp. and Bifidobacterium spp. of the mixtures (F_1 and F_2) increased during the times of treatment until 60 days in the probiotic groups, with respect to the placebo group (F_3). All the species included in the formulations remained in the gut also 30 days after the follow-up from the last ingestion, except for Bifidobacterium. Our results confirmed data reported by Kajander et al. These authors demonstrated that all supplemented strains remained stable during the treatment, with the exception of Bifidobacterium species which decreased after treatments with a multispecies probiotic mixture [14].

Moreover, in our study, significant differences in the number of responders to the severity of symptoms were recorded between the two probiotic mixtures, F_1 and F_2, with respect to placebo $F_{\_} 3$ group $(P<0.001)$. No significant differences were registered between F_1 and F_2 $(P>0.05)$, and the effects of both of them are significant when compared to their respective baselines. These data are in agreement with previous data from multispecies probiotics treatment of IBS subjects $[14,15]$. Compared with placebo, probiotic groups F_1 and F_2 were effective for the primary efficacy endpoints of the study as well as for the secondary endpoints, that is, the maintaining of the obtained effects 30 days after the last product intake. The change of symptoms is correlated to the improvement in the quality of life and resulted in being significantly higher in the probiotic groups compared with the placebo group. Although a great number of data deriving from literature $[16-18,30]$ indicate that probiotics may be helpful in the treatment of IBS symptoms, in particular with respect to constipation, their conclusions vary because of inadequate sample size, type of study design, and use of various probiotic strains. These data are in agreement with our data concerning the improvement of specific probiotic treatment versus placebo (specifically in patients with IBSC) for some of the endpoints, improving symptoms such as pain, flatulence, and bloating, but not others (transit time and urgency and abdominal cramps) [30]. Moreover, in most of the reported cases, the decrease in constipation frequency score was approximately twofold greater in the probiotic groups than in the placebo groups and these results are in line with data deriving from our clinical study.

Thus, the clinical improvement of this study may be associated with the maintenance (species-specific) of the compositional stability of the intestinal microbiota from probiotics consumption and with their positive effects in subjects affected by irritable bowel syndromes.

\section{Conclusions}

In conclusion, the different species of probiotics administered to the IBS-C subjects determine a cooccurrence between the changes in the analysed probiotic groups and an improvement of IBS-C symptoms. This study represents the development of a clinical trial that can support the role of intestinal bacteria in the IBS diseases and the potential role of probiotics belonging to various species in the management of these disorders. 


\section{Ethical Approval}

All procedures performed in studies involving human participants were in accordance with the ethical standards of the institutional and/or national research committee and with the 1964 Helsinki declaration and its later amendments or comparable ethical standards.

\section{Consent}

Informed consent was obtained from all individual participants included in the study.

\section{Competing Interests}

All the authors declare that they have no conflict of interests.

\section{Acknowledgments}

The research was conducted through the Probioplus4Food Project no. 30221122, funded by MIUR and Lombardy Region, Italy. The authors thank Principium Europe Srl. for supplying the bacterial strains and Farcoderm for the recruited subjects of this study.

\section{References}

[1] P. B. Eckburg, E. M. Bik, C. N. Bernstein et al., "Microbiology: diversity of the human intestinal microbial flora," Science, vol. 308, no. 5728, pp. 1635-1638, 2005.

[2] S. K. Mazmanian, H. L. Cui, A. O. Tzianabos, and D. L. Kasper, "An immunomodulatory molecule of symbiotic bacteria directs maturation of the host immune system," Cell, vol. 122, no. 1, pp. 107-118, 2005.

[3] V. R. Alonso and F. Guarner, "Linking the gut microbiota to human health," British Journal of Nutrition, vol. 109, supplement 2, pp. S21-S26, 2013.

[4] G. Tomasello, M. Bellavia, V. D. Palumbo, M. C. Gioviale, P. Damiani, and A. I. L. Monte, "From gut microflora imbalance to mycobacteria infection: is there a relationship with chronic intestinal inflammatory diseases?" Annali Italiani di Chirurgia, vol. 82, no. 5, pp. 361-368, 2011.

[5] F. Guarner and J.-R. Malagelada, "Gut flora in health and disease," The Lancet, vol. 361, no. 9356, pp. 512-519, 2003.

[6] J. Mättö, L. Maunuksela, K. Kajander et al., "Composition and temporal stability of gastrointestinal microbiota in irritable bowel syndrome-a longitudinal study in IBS and control subjects," FEMS Immunology and Medical Microbiology, vol. 43, no. 2, pp. 213-222, 2005.

[7] C. Abraham and J. H. Cho, "Mechanisms of disease: inflammatory bowel disease," The New England Journal of Medicine, vol. 361, no. 21, pp. 2066-2078, 2009.

[8] M. Bellavia, G. Damiano, M. C. Gioviale et al., "Abnormal expansion of segmented filamentous bacteria in the gut: a role in pathogenesis of chronic inflammatory intestinal diseases?" Reviews in Medical Microbiology, vol. 22, no. 3, pp. 45-47, 2011.

[9] M. Bellavia, G. Tomasello, M. Romeo et al., "Gut microbiota imbalance and chaperoning system malfunction are central to ulcerative colitis pathogenesis and can be counteracted with specifically designed probiotics: a working hypothesis," Medical
Microbiology and Immunology, vol. 202, no. 6, pp. 393-406, 2013.

[10] W. Strober, I. Fuss, and P. Mannon, "The fundamental basis of inflammatory bowel disease," Journal of Clinical Investigation, vol. 117, no. 3, pp. 514-521, 2007.

[11] M. Llopis, M. Antolin, M. Carol et al., "Lactobacillus casei downregulates commensals' inflammatory signals in Crohn's disease mucosa," Inflammatory Bowel Diseases, vol. 15, no. 2, pp. 275-283, 2009.

[12] M. Pammi, A. Flores, M. Leeflang, and J. Versalovic, "Molecular assays in the diagnosis of neonatal sepsis: a systematic review and meta-analysis," Pediatrics, vol. 128, no. 4, pp. e973-e985, 2011.

[13] M. Venkatesh, A. Flores, R. A. Luna, and J. Versalovic, "Molecular microbiological methods in the diagnosis of neonatal sepsis," Expert Review of Anti-Infective Therapy, vol. 8, no. 9, pp. 10371048, 2010.

[14] K. Kajander, E. Myllyluoma, M. Rajilić-Stojanović et al., "Clinical trial: multispecies probiotic supplementation alleviates the symptoms of irritable bowel syndrome and stabilizes intestinal microbiota," Alimentary Pharmacology and Therapeutics, vol. 27, no. 1, pp. 48-57, 2008.

[15] J. S. Yoon, W. Sohn, O. Y. Lee et al., "Effect of multispecies probiotics on irritable bowel syndrome: a randomized, doubleblind, placebo-controlled trial," Journal of Gastroenterology and Hepatology, vol. 29, no. 1, pp. 52-59, 2014.

[16] A. P. S. Hungin, C. Mulligan, B. Pot et al., "Systematic review: probiotics in the management of lower gastrointestinal symptoms in clinical practice-an evidence-based international guide," Alimentary Pharmacology and Therapeutics, vol. 38, no. 8, pp. 864-886, 2013.

[17] A. Agrawal, L. A. Houghton, J. Morris et al., "Clinical trial: the effects of a fermented milk product containing Bifidobacterium lactis DN-173 010 on abdominal distension and gastrointestinal transit in irritable bowel syndrome with constipation," Alimentary Pharmacology and Therapeutics, vol. 29, no. 1, pp. 104-114, 2009.

[18] S. Guglielmetti, D. Mora, M. Gschwender, and K. Popp, "Randomised clinical trial: Bifidobacterium bifidum MIMBb75 significantly alleviates irritable bowel syndrome and improves quality of life-a double-blind, Placebo-Controlled Study," Alimentary Pharmacology and Therapeutics, vol. 33, no. 10, pp. 1123-1132, 2011.

[19] I. Presti, G. D'Orazio, M. Labra et al., "Evaluation of the probiotic properties of new Lactobacillus and Bifidobacterium strains and their in vitro effect," Applied Microbiology and Biotechnology, vol. 99, no. 13, pp. 5613-5626, 2015.

[20] I. Aloisio, C. Santini, B. Biavati et al., "Characterization of Bifidobacterium spp. strains for the treatment of enteric disorders in newborns," Applied Microbiology and Biotechnology, vol. 96, no. 6, pp. 1561-1576, 2012.

[21] D. A. Drossman, E. Corazziari, M. Delvaux et al., Eds., Rome III: The Functional Gastrointestinal Disorders, Degnon Associates, McLean, Va, USA, 3rd edition, 2006.

[22] D. L. Patrick, D. A. Drossman, I. O. Frederick, J. Dicesare, and K. L. Puder, "Quality of life in persons with irritable bowel syndrome: development and validation of a new measure," Digestive Diseases and Sciences, vol. 43, no. 2, pp. 400-411, 1998.

[23] S. J. Lewis and K. W. Heaton, "Stool form scale as a useful guide to intestinal transit time," Scandinavian Journal of Gastroenterology, vol. 32, no. 9, pp. 920-924, 1997. 
[24] M. Enrico, Biological sciences [Ph.D. thesis], The University of Milano-Bicocca, 2015.

[25] M. V. Matz, R. M. Wright, and J. G. Scott, "No control genes required: bayesian analysis of qRT-PCR data," PloS ONE, vol. 8, no. 8, Article ID e71448, 2013.

[26] H. Wickham, ggplot2: Elegant Graphics for Data Analysis, Springer Science \& Business Media, Springer-Verlag, New York, NY, USA, 2009.

[27] J. Plaza-Diaz, C. Gomez-Llorente, L. Fontana, and A. Gil, "Modulation of immunity and inflammatory gene expression in the gut, in inflammatory diseases of the gut and in the liver by probiotics," World Journal of Gastroenterology, vol. 20, no. 42, pp. 15632-15649, 2014.

[28] A. P. M. Kerckhoffs, M. Samsom, M. E. van der Rest et al., "Lower Bifidobacteria counts in both duodenal mucosaassociated and fecal microbiota in irritable bowel syndrome patients," World Journal of Gastroenterology, vol. 15, no. 23, pp. 2887-2892, 2009.

[29] E. Malinen, T. Rinttilä, K. Kajander et al., "Analysis of the fecal microbiota of irritable bowel syndrome patients and healthy controls with real-time PCR," American Journal of Gastroenterology, vol. 100, no. 2, pp. 373-382, 2005.

[30] P. Moayyedi, A. C. Ford, N. J. Talley et al., "The efficacy of probiotics in the treatment of irritable bowel syndrome: a systematic review," Gut, vol. 59, no. 3, pp. 325-332, 2010. 


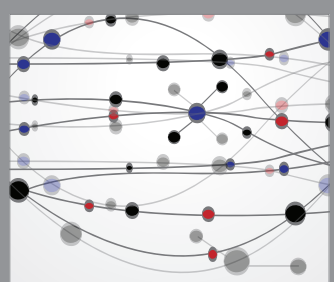

The Scientific World Journal
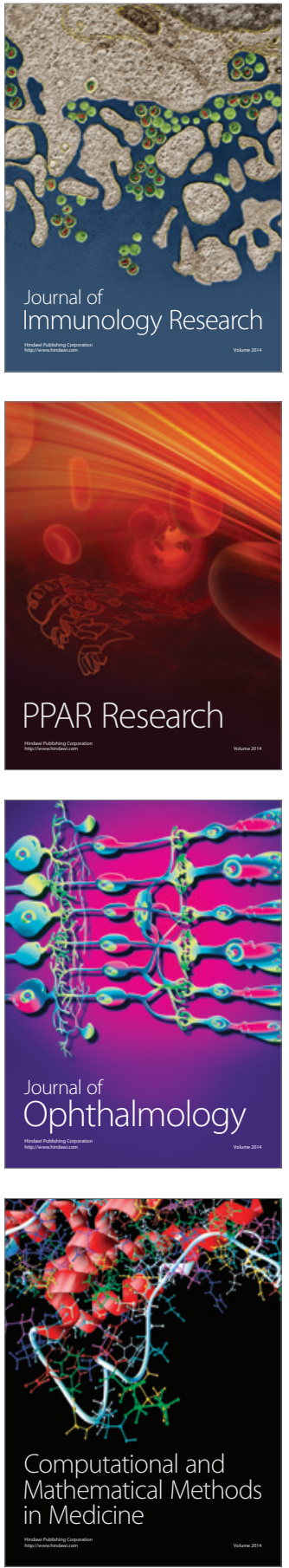

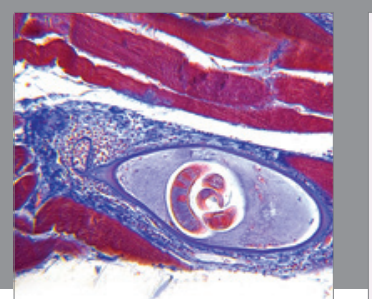

Gastroenterology Research and Practice

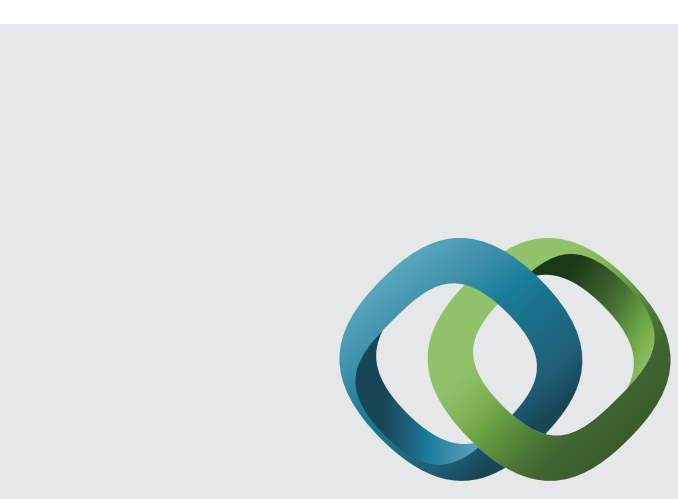

\section{Hindawi}

Submit your manuscripts at

http://www.hindawi.com
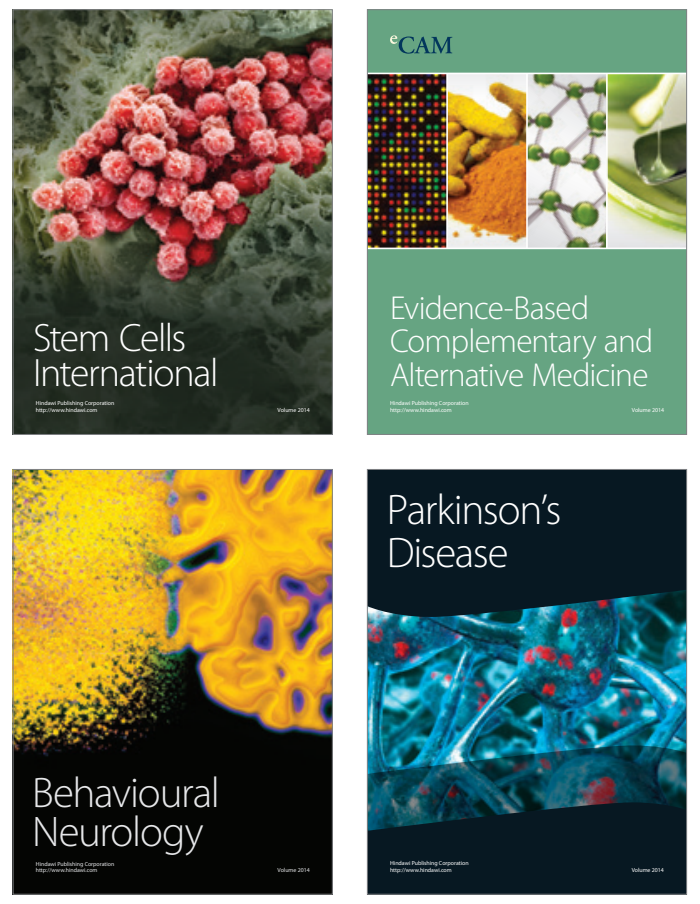
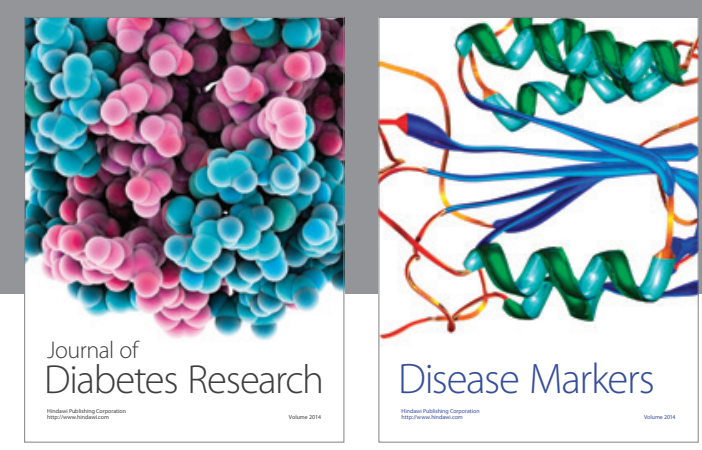

Disease Markers
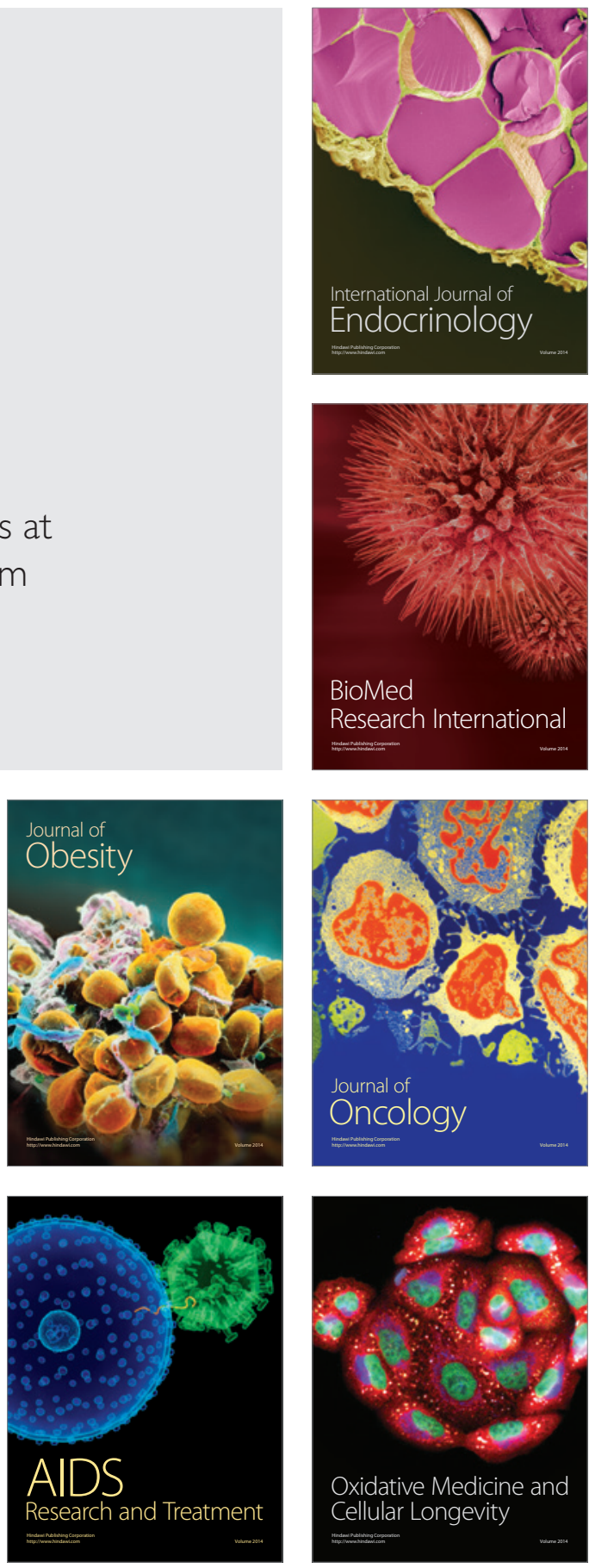